\title{
Local Mathematics and No Information at a Distance; Some Effects on Physics and Geometry ${ }^{+}$
}

\author{
Paul Benioff \\ Argonne national laboratory, Argonne, IL 60439, USA; pbenioff@anl.gov \\ + Conference Theoretical Information Studies, Berkeley, CA, USA, 2-6 June 2019.
}

Published: 19 May 2020

check for updates

\begin{abstract}
Local mathematics consists of a collection of mathematical systems located at each space and time point. The collection is limited to the systems that include numbers in their axiomatic description. A scalar map between systems at different locations is based on the distinction of two conflated concepts, number and number value. The effect that this setup has on theory descriptions of physical and geometric systems is described. This includes a scalar spin 0 field in gauge theories, expectation values in quantum mechanics and path lengths in geometry. The possible relation of the scalar map to consciousness is noted.
\end{abstract}

Keywords: local mathematics; information; distance; physics; geometry

\section{Introduction}

Local mathematics and no information at a distance affect [1] theoretical descriptions of systems in physics and geometry. The use of local mathematics as the basis for describing physics and geometry is an extension to many different types of mathematical systems of the use of separate vector spaces [2] at each point of space time for the description of gauge theories. The relation between states in the separate vector spaces is determined by properties of a map between vector spaces at different locations. The need for a map is based on the observation [3] that the meaning of a state in a vector space at one location does not determine the meaning of the same state in a vector space at another location. The no information at a distance principle $[2,4]$ as used in gauge theories is an equivalent statement of this observation.

This work extends these ideas to separate scalar fields (real or complex numbers) associated with the vector spaces at different space time locations. The existence of a map between number structures similar to that between vector spaces is based on the no information principle and distinction between two conflated but distinct, concepts, number and number value.

Local mathematics consists of the further extension of these concepts to the localization of all types of mathematical systems that include scalars in their axiomatic description. All types of numbers, natural numbers, integers, rational, real, and complex numbers, vector spaces, algebras, group representations, geometric spaces, etc. are included. Local mathematics and no information at a distance provide the arena for the description of the relation between physical and geometric systems and their theoretical descriptions.

\section{Number and Number Value}

The distinction between number and number value is easiest to see for the natural numbers, $0,1,2,3,4, \ldots$ These constitute a set of numbers that, along with addition and multiplication operations and an order relation and the axioms of arithmetic, form a natural number structure, $\bar{N}_{1}$. In this structure the number 0 has value 0 , the number 1 has value 1 , the number 2 has value 2 , and so on. 
The set of even numbers, $0,2,4,6, \ldots$ also forms a structure for the natural numbers provided the multiplication operation in this structure, $\bar{N}_{2}$ relative to that in $\bar{N}_{1}$ is scaled by $1 / 2$. That is $\times_{2}=1 / 2 \times_{1}$.

In $\bar{N}_{2}$ the value of 0 is 0 , the value of 2 is one, The value of 4 is 2, etc. This shows that number and number value are distinct. Extension to the set of every $n$th number, $0, n, \ldots$ as in $\bar{N}_{n}$, shows that the value of the number $n$ in this structure is 1 . If $n=4$ then the number $n$ also has value 2 in $\bar{N}_{2}$ and value 4 in $\bar{N}_{1}$. A good example of the multiplicity of values is given by the number 30 . One has

$$
30_{1}=15_{2}=10_{3}=6_{5}=5_{6}=3_{10}=2_{15}=1_{30} .
$$

This represents the fact that the number 30 has value 15 in $\bar{N}_{2}$ has value 5 in $\bar{N}_{6}$, etc.

Number scaling extends to the rational, real, and complex number structures bringing them to the scope of non-Diophantine arithmetics [5]. The number that has value, $a$ in the real number structure, $\bar{R}_{r}$, has value $(r / s) a$ in the real number structure, $\bar{R}_{s}$. A convenient way to write numbers in different structures is as a value with a scaling subscript. For example $a_{r}$ denotes the number with value $a$ in $\bar{R}_{r}$. The representation of this same number in $\bar{R}_{s}$ is $[(r / s) a]_{s}$.

The final step is the extension of the use of scaling factors to a real valued field of scaling factors over a base space and time, $T \times M$. Let $\alpha(x, t)$ be the value of the field at space and time points, $x, t$. The scaling factor $g(x, t)$ is defined by

$$
g(x, t)=e^{\alpha(x, t)} .
$$

This leads to local mathematics. At each point in $T \times M$, there is a collection, $\{S\}_{g(x, t)}=\bigcup_{S} S_{g(x, t)}$, of all mathematical system types that use numbers in their axiomatic description. Included are number structures of all types, such as rational numbers, $\bar{R} a_{g(x, t)}$, real numbers, $\bar{R}_{g(x, t)}$, complex numbers, $\bar{C}_{g(x, t)}$, vector spaces, $\bar{V}_{g(x, t)}$, etc. Figure 1 illustrates the location of local mathematics at different points in the base space and time.

From now on the scaling field will be referred to as a value or meaning field. This represents the true meaning of the field as it determines the meaning or value of numerical quantities and properties in the other types of systems in the local mathematics. An example is the length of vectors in vector spaces.

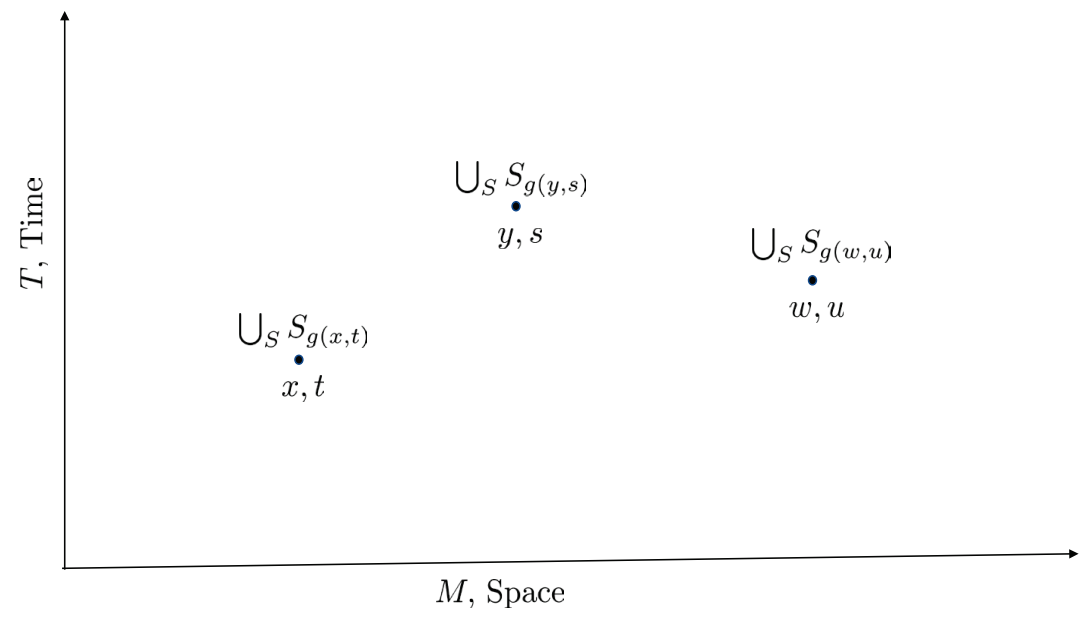

Figure 1. Illustration of the existence of local mathematics at each point in space and time. The illustration is for three locations, $x, t, y, s$, and $w, u$.

\section{Effect of the No Information at a Distance Principle}

Theoretical physics is concerned with the construction of models of systems and events occurring in space and time or in space time. The entities appearing in theory include the meaning of numerical, vector and other types of physical quantities. If $a$ is a numerical quantity in theoretical physics, the number with meaning $a$ in a number structure at location $x, t$ is denoted by $a_{g(x, t)}$. 
One would like to use the value of the number $a_{g(x, t)}$ to determine the value of the same number at a different space and time location, $y, s$. This is forbidden by the no information at a distance principle. The value, $a$ of the number $a_{g(x, t)}$ does not determine the value of the same number at $y, s$.

This problem is solved by the use of a number preserving number value changing map between numbers in the local mathematics at different locations. These maps, as connections, implement the parallel transports of number meanings from one location to another. ${ }^{1}$

Let $b$ be a real or complex number whose meaning is $a$ in the local mathematics at $x, t$. This number is represented in the local mathematics as $a_{g(x, t)}$. Parallel transport of this number to the local mathematics at $y, s$ is represented by

$$
a_{g(x, t)} \rightarrow C_{g}(y, s ; x, t) a_{g(x, t)}=\left[e^{-\alpha(y, s)+\alpha(x, t)} a\right]_{g(y, s)} .
$$

Here $C_{g}(y, s ; x, t)$ is the connection or parallel transport map. Equation (2) has been used to replace the $g$ function by its exponential form.

Equation (3) holds if the number, $a$ is replaced by a vector $\vec{v}$. This shows that the effect of parallel transport is to change the length of the vector. The direction is unchanged.

\section{Theoretical Description of Physical and Geometric Quantities}

The effect of the meaning field shows up in theoretical descriptions of physical quantities represented by derivatives or integrals over space and or time. Examples of such quantities include path lengths, Expectation values in quantum mechanics, and derivatives of fields appearing in field theories.

As a specific example, let $\psi$ be a theoretical model of a real scalar field over space. The field derivative $d \psi(x) / d x$, is not defined in the presence of local mathematics. The reason is that the definition of the derivative,

$$
\frac{d \psi(x)}{d x}=\lim _{x \rightarrow 0} \frac{[\psi(x+d x)]_{g(x+d x)}-\psi(x)_{g(x)}}{d x}
$$

is not defined. Arithmetic comparison of numbers in structures at different locations is not defined. It is limited to comparison between numbers with values in a structure at a single location.

This problem is fixed by parallel transport of $[\psi(x+d x)]_{g(x+d x)}$ to $\left[e^{\alpha(x+d x)-\alpha(x)} \psi(x+d x)\right]_{g(x)}$. Use of a Taylor expansion to simplify the exponent and exponential gives the definition of the defined derivative as

$$
\left[D_{x} \psi(x)\right]_{g(x)}=\left[\left(\frac{d}{d x}+A(x)\right) \psi(x)\right]_{g(x)} .
$$

Here $^{2} A(x)=d \alpha(x) / d x$.

The same problem appears in integrals over the space variable, $x$. With local mathematics as the background, the integrand $I(x)$ of the integral, $\int I(x) d x$, becomes $[I(x)]_{g(x)}$. The summation of the integrand over the integration variable, implied in the definition of the integral over $x$, is not defined.

This is fixed by parallel transport of the integrand to a reference location, $z$. The integrand becomes $\left[e^{-\alpha(z)+\alpha(x)} I(x)\right]_{g(z)}$. The price for obtaining a defined integral is inclusion of the extra $x$ dependent factor, $e^{\alpha(x)}$ with the integrand. The constant factor, $e^{-\alpha(z)}$, is a normalization factor.

Gauge theories provide one example of this effect. The term. $U(y, y+d y) \psi(y+d y)_{g(y+d y)}$ in the definition of the derivative in gauge theory Lagrangians is expanded to $C_{g}(y, y+d y) U(y, y+d y) \psi(y+$ $d y)_{g(y, d y)}$. The covariant derivative becomes

Parallel transport of a number meaning is not the same as the effect on meaning resulting from the physical translation of a
number from one location to another. number from one location to another.
This same method is used to define covariant derivatives in gauge theories. 


$$
\left[D_{v, y} \psi(y)\right]_{g(y)}=\left[\left(\frac{d}{d y^{v}}+b A_{v}(y)+\ldots\right) \psi(y)\right]_{g(y)} .
$$

The $+\ldots$ denotes the field for the bosons in physics. The $A$ field denotes the gradient of the scalar field $\alpha$, presumably of spin 0 , any mass is possible.

The Higgs field is the only scalar spin 0 field in particle physics. Is the $\alpha$ field the Higgs field? The $\alpha$ field interacts with all fermion fields. It appears in all Lagrangians with field derivatives.

Quantum mechanics and geometry provide many examples of the effect of the $\alpha$ field. Examples include the normalization integral, $\int|\psi(y)|^{2} d y$ and path length integral, $\int \mid d \vec{p}(s) d s$. These integrals are defined provided extra factors, $e^{\alpha(y)-\alpha(x)}$ and $e^{\alpha(p(s), s)-\alpha(x, t)}$ are added to the integrand. One gets $\left[\int e^{\alpha(y)-\alpha(x)}|\psi(y)|^{2} d y\right]_{g(x)}$ and $\left[\int e^{\alpha(p(s), s)-\alpha(x, t)}|d \vec{p}(s)| d s\right]_{g(x, t)}$. Here $p$ is a path parameterized by the tine $s . x$ and $x, t$ denote reference locations in space and in space and time.

\section{Restrictions on the $\alpha$ Field}

The lack of experimental evidence for the presence of the $\vec{A}$ potential puts restrictions on the space and time variation of the $\alpha$ field. Since experiments are implemented by us as conscious observers, the restrictions on the $\alpha$ field are limited to a region of cosmological space and time that is or can be occupied by us. This region includes the solar system and observers around stars within effective two way communication distance from us. One literature estimate [6] of the region size includes the sun and stars less than 1000 lightyears distant. The exact size is not important. It should be a very small fraction of the universe. Outside this region there are no restrictions on the variation of the $\alpha$ field.

Experiments are local in that the system prepared for measurement is local. Measurements are local, but the system whose properties are to be measured is taken as is. These are systems that are too large or too far away. Examples include planets or the sun and other stars.

\section{The Basic Role of the $\alpha$ Field}

One begins with basic fact that all physical systems are systems in various states. As such they have no intrinsic meaning at all. Meaning is provided by a connection of the physical systems to mathematical theoretical models of the systems and their dynamics. The $\alpha$ field provides this connection. Its presence is a necessary condition for the existence of theoretical models or descriptions of physical systems and their dynamics. Without the meaning field the world of physical systems and their dynamics would be meaningless.

The connection [7] to consciousness arises because meaning is a basic property of consciousness. The $\alpha$ field provides a connection between physical systems and consciousness. The existence of the $\alpha$ field, independent of dependent on space and/or time, is a necessary component of consciousness. We, as conscious observers, provide meaning to otherwise meaningless physical systems and their dynamics. The meaning consists of theoretical models of physical systems. The theoretical models use the local mathematics at the locations of each of us. ${ }^{3}$

\section{Conclusions}

This work begins with the extension of the use in gauge theories of separate vector spaces and the no information at a distance principle. This is extended to include numbers and all mathematical systems including numbers in their description. This local mathematics, the observation that number and number meaning or value are distinct, and the application of the no information at a distance principle to this distinction are briefly described. It is seen that the resulting number value or meaning

3 The distinction between meaningless physical systems and theoretical models is already present in the distinction between syntactic and semantic systems in mathematical logic. 
field has an effect on theoretical descriptions of physical and geometric systems. The connection to consciousness is also noted.

Funding: This research received no external funding.

Conflicts of Interest: The author declares no conflicts of interest.

\section{References}

1. Benioff, P. The no information at a distance principle and local mathematics: Some effects on physics and geometry. In Theoretical Information Studies; Burgin, M., Dodig-Crnkovic, G., Eds.; World Scientific Press: Singapore, 2020.

2. Montvay, I.; Münster, G. Quantum Fields on a Lattice; Cambridge University Press: Cambridge, UK, 1994; Chapter 3.

3. Yang, C.N; Mills, R.L. Conservation of Isotopic Spin and Isotopic Gauge Invariance. Phys. Rev. 1954, 96, 191-195.

4. Mack, G. Physical principles, geometrical aspects, and locality properties of gauge field theories. Fortshritte der Physik 1981, 29, 135-185.

5. Burgin, M. Elements of Non-Diophantine Arithmetics. In Proceedings of the 6th Annual International Conference on Statistics, Mathematics and Related Fields, Honolulu, HI, USA, 17-19 January 2007; pp. 190-203

6. Smith, H.A. Alone in the Universe. Am. Sci. 2011, 99, 320. doi:10.1511/2011.91.320

7. Benioff, P. Relation between observers and effects of number valuation in science. J. Cogn. Sci. 2018, 19, $229-251$.

(C) 2020 by the author. Licensee MDPI, Basel, Switzerland. This article is an open access article distributed under the terms and conditions of the Creative Commons Attribution (CC BY) license (http:/ / creativecommons.org/licenses/by/4.0/). 